\title{
Sarcoma of the Ewing Family in Pregnancy: A Case Report of Intrauterine Fetal Death after Induction of Chemotherapy
}

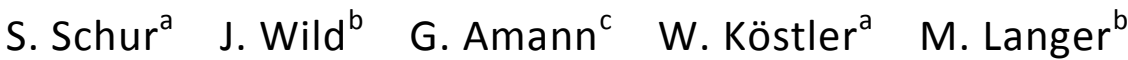 \\ T. Brodowicz ${ }^{a}$ \\ ${ }^{a}$ Comprehensive Cancer Center - Musculoskeletal Tumors, GIST, Bone and Soft \\ Tissue Sarcoma Program, Clinical Division of Oncology, Department of Internal \\ Medicine I, Sarcoma Platform Austria, ${ }^{b}$ Clinical Division of Obstetrics and \\ Feto-Maternal Medicine, Department of Obstetrics and Gynecology, and \\ ${ }^{c}$ Clinical Institute of Pathology, Medical University of Vienna, General Hospital \\ Vienna, Vienna, Austria
}

\section{Key Words}

Ewing's sarcoma - Pregnancy - Neoadjuvant chemotherapy - Intrauterine fetal death . Multidisciplinary team approach

\begin{abstract}
Ewing's sarcoma is an ultra-orphan disease (2/1,000,000/year) which requires a multimodal therapy approach in high-volume centers. Treatment consists of pre-operative therapy followed by surgery and post-operative combination of chemo-radiotherapy. Experience with diagnosis and therapy of Ewing's sarcoma in pregnancy is very limited. We herein report the case of an atypical Ewing's sarcoma detected in the second trimester of gestation. Neoadjuvant chemotherapy was initiated and resulted in substantial tumor shrinkage and intrauterine fetal death. The rare nature of this condition underlines once more the need for a multidisciplinary team to improve the quality of care for this highly special patient collective.
\end{abstract}

\section{Background}

According to recent literature, approximately 1 in 1,000 term pregnancies is complicated with cancer. This incidence is increasingly encountered in clinical practice with the rising trend of postponing pregnancy to later in life. Breast cancer is the most common tumor treated during gestation followed by ovarian cancer, cervical cancer, 
leukemia, lymphoma, and lung cancer [1,2]. Bone and soft tissue sarcomas, being generally rare diseases, present more infrequently during gestation [3, 4]. In total, 12 patients with sarcoma [5-16] and 1 with a primitive neuroectodermal tumor [17] have been treated with chemotherapy while being pregnant. However, in total, only 5 women have been described who were primarily diagnosed and treated with Ewing's sarcoma during pregnancy $[9,10,13,14,17]$.

Based on the prevailing literature, we herein report the 6th case of a woman diagnosed and treated with Ewing's sarcoma in the second trimester of gestation and emphasize once more the need for a multidisciplinary team approach to improve the quality of care for this highly special patient collective.

\section{Case Report}

A 27-year-old primiparous Caucasian woman recognized a rapidly growing mass at the right proximal mediodorsal lower limb in the 18th gestational week and was first admitted in midDecember 2011 to an outward medical department. It was the patient's first pregnancy and she had no prior medical history. Magnetic resonance imaging (MRI) of the small pelvis showed an $11 \times 18 \mathrm{~cm}$ big lesion with considerable dislocation of the vagina and diffuse tumorous infiltration of the uterus harboring the fetus (fig. 1). Furthermore, a CT scan revealed pulmonary metastasis. A biopsy of the pelvic tumor was performed, revealing round, blue sarcoma cells. Distinct immunostaining for CD99 was positive, whereas no other antibody connection with specific differentiation reacted positively (e.g. desmin, pancytokeratin, EMA, GFAP, and S100). Polymerase chain reaction investigation for specific translocations included $\mathrm{t}(9 ; 22), \mathrm{t}(9 ; 17)$ (extraskeletal myxoid chondrosarcoma), $\mathrm{t}(11 ; 22)$, $\mathrm{t}(21 ; 22)$ (Ewing's sarcoma), and $\mathrm{t}(\mathrm{X} ; 18)$ (synovial sarcoma) and revealed no positive results. In addition, FISH investigations proved the integrity of ESWR-1 and FUS gene, excluding the involvement in translocations, so that the final diagnosis of an atypical Ewing's sarcoma was made. A gynecological assessment proved an intact gestation. At that time, a termination of pregnancy by medication or fetocide with following vaginal birth or cesarean section would not have been possible without high risk for the patient because of diffuse tumorous infiltration of the uterus, compression of the vagina, and dislocation of the cervix (fig. 2). Hence, only the initiation of an aggressive multi-agent chemotherapy seemed reasonable, and the patient was transferred to the Department of Oncology at the Medical University of Vienna. A gynecological examination showed an ulcerated mass between the right labium majus and the anal region as well as a left-sided dislocation and constriction of the vagina with a non-palpable portio. However, the fetus was vital and the amniotic fluid in normal range. After extensive interdisciplinary consultation of the mother's as well as the fetus's prognosis, a neoadjuvant polychemotherapy according to the Euro Ewing-08 protocol (VIDE scheme) was initiated. It consisted of etoposide $\left(150 \mathrm{mg} / \mathrm{m}^{2} /\right.$ day $)$, ifosfamide $\left(3,000 \mathrm{mg} / \mathrm{m}^{2} /\right.$ day $)$, and doxorubicin $\left(20 \mathrm{mg} / \mathrm{m}^{2} /\right.$ day) on days $1-3$ as well as vincristine $(2 \mathrm{mg} /$ day) on day 1 starting in the gestational week 18+6. Under adequate antiemetic prophylaxes and bone marrow support, chemotherapy was well tolerated by the patient. At the end of the 20st gestational week, an ultrasound examination was performed, showing an oligohydramnion but vital fetus with regular cardiac activity. MRI after the first cycle of chemotherapy attested a partial response (70\% tumor shrinkage). Before the beginning of the second cycle of VIDE chemotherapy in gestational week 23, once more fetal monitoring had proven a vital gravidity with persistent oligohydramnion. In gestational week $23+5$, the second cycle was administered and again well tolerated. On day 3 of the second VIDE cycle (gestational week $23+7$ ), the patient complained about spasmodic lower abdominal pain. An ultrasound examination revealed a negative fetal cardiac activity consistent with intrauterine fetal death. A gynecological examination was performed showing no signs of tumor infiltration to the vagina and a firm, closed portio with no visible bleeding. Decision in favor of vaginal birth was made and induction of labor with mifepristone and misoprostol was started. In gestational week $24+1$, abortion occurred, followed by an uncomplicated postpartum course. Subsequently, the patient received 4 further cycles of chemotherapy according to the Euro Ewing-08 protocol (VIDE scheme). During chemotherapy cycle 4, a stem cell harvest was accomplished. Five weeks after the restaging, definitive surgical tumor resection was performed including pararectal as well as paravaginal incision and hypogastric laparotomy. Postoperative histology confirmed the primary diagnosis. Subsequently, the patient 
received 1 cycle of adjuvant chemotherapy according to the VAC scheme consisting of cyclophosphamide $\left(1,500 \mathrm{mg} / \mathrm{m}^{2} /\right.$ day $)$ and actinomycine $\mathrm{D}\left(0.75 \mathrm{mg} / \mathrm{m}^{2} /\right.$ day $)$ on day 1 and 2 , and vincristine ( $2 \mathrm{mg} /$ day) on day 1 , which was well tolerated. The most recent restaging showed no evidence of disease.

Written informed consent was obtained from the patient for publication of this case report and any accompanying images.

\section{Discussion}

Lysyj and Bergquist [13] reported the first case of Ewing's sarcoma in pregnancy in 1963. Their patient presented at the 32nd week of gestation with pain in the right leg and was diagnosed with Ewing's sarcoma of the pubic ramus. Cesarean section was performed in week 36 of gestation. Neither chemotherapy nor radiation therapy was administered during gestation [13]. In the second case, a 21-year-old patient was diagnosed with Ewing's sarcoma of the left iliac wing in gestational week 25. She was treated with 1 cycle of multi-agent chemotherapy consisting of actinomycine D, cyclophosphamide, bleomycin, vincristine, and doxorubicin before she delivered a healthy baby by cesarean section in gestational week 34 [14]. The third case report by Merimsky et al. [10] describes the case of an Ewing's sarcoma of the right sacroiliac bone in a childbearing woman. Starting in the 27th week of pregnancy, 3 courses of 3weekly doses of doxorubicin plus ifosfamide and mesna (ADR-IFX) were administered. Cesarean section had been pre-planned after completion of 3 cycles of chemotherapy and after achieving fetal lung maturation. The outcome was a small but normally developed baby [10]. Nakajima et al. [9] report the case of a 17-year-old woman with an extraskeletal Ewing's sarcoma of the left upper leg who was treated with a combination of doxorubicin and ifosfamide during the 25th to 30th week of pregnancy. After 3 cycles of chemotherapy, mild intrauterine growth retardation of the baby occurred and it was electively delivered in the 32 nd week by cesarean section. The baby was small for gestational age and needed to be intubated because of irregular respiratory effort. Additionally, it received phototherapy for hyperbilirubinemia and erythropoietin because of a decrease in hemoglobin and reticulocytes. However, further on, the baby was growing adequately and had no known abnormalities at 8 months of age [9]. The 5th case report by Ateser et al. [17] describes the case of a primitive neuroectodermal tumor (PNET) of the ovary diagnosed in the 24th week of gestation. Starting in gestational week 30, 2 cycles of chemotherapy consisting of doxorubicin, cyclophosphamide, and vincristine were administered and a healthy baby was delivered by cesarean section in gestational week 37. After pregnancy, the mother was found to have metastatic disease and died due to progressive disease 13 months after the initial diagnosis [17].

When comparing our patient with the other case reports described above, the other women diagnosed with Ewing's sarcoma delivered safely. In this respect, it may be of interest that our patient received chemotherapy earlier in the course of gestation (in the 20th week), while in the other patients, chemotherapy was administered starting in week 25 the earliest. Comparable to our case report, most of the chemotherapy regimens were doxorubicin based. The multi-agent chemotherapy protocol administered in our patient consisted of ifosfamide, doxorubicin and vincristine and, unlike in other reported cases, etoposide. Nevertheless, the use of etoposide is 
documented in at least 25 sarcoma cases with a constantly favorable outcome for the offspring [18].

In every pregnant woman in whom a sarcoma is suspected, the diagnostic and therapeutic approaches have to be even more specifically tailored. Surgical procedures, such as open biopsy, tumor resection, and limb-sparing surgery, are regarded as relatively safe during gestation [4]. The risk of chemotherapy during pregnancy depends on the drugs used and the gestational age of the fetus. Most of the information on chemotherapy in pregnancy stems from series that used multi-agent protocols, and therefore it is difficult to separate the effects of individual drugs on the fetus [3]. In osteosarcoma and Ewing's sarcoma, neoadjuvant chemotherapy is well accepted and should be started whenever possible during gestation. Radiation therapy is regarded as harmful because of the inability to shield the fetus from ionizing scatter. Therefore, it should be avoided in pregnant women and delayed until after delivery [2, 3]. In cases where the tumor and the fetus share the same limited space in the pelvis or abdominal cavity, premature labor might occur with a small-sized newborn, which is not related exclusively to chemotherapy-associated growth retardation. Hence, cesarean section may be the preferred way of giving birth when the primary tumor affects the pelvic bones and structures [3].

The care of a pregnant woman diagnosed with cancer involves evaluation of competing maternal and fetal risks and benefits. Furthermore, the diagnosis of a malignant disease during pregnancy raises serious medical as well as ethical, emotional, religious, and philosophical questions. Hence, the mother's well-being and the fetal risk by introducing early diagnosis and therapy should always be weighed against the risk to the mother's health and fetal well-being in carrying on an uninterrupted gestation. From our point of view, the curability of the mother's disease is the most important indication. Thus, in the case of a favorable prognosis or curative situation, chemotherapy should be given as early as possible.

Despite lack of robust consensus, multi-agent chemotherapy should be instituted early in the course of malignant sarcomas since the risk of metastasis occurrence is high. However, because rare scenarios like this raise major medical and ethical dilemmas, therapy should be tailored to each case and only be performed in specialized high-volume centers as the subject of a multidisciplinary consultation.

\section{Acknowledgement}

We want to thank Christopher Fletcher, Chief of Oncopathology at the Dana-Farber Cancer Institute, for his support in reconfirming the pathologic diagnosis.

\section{Disclosure Statement}

The authors declare that they have no competing interests. 


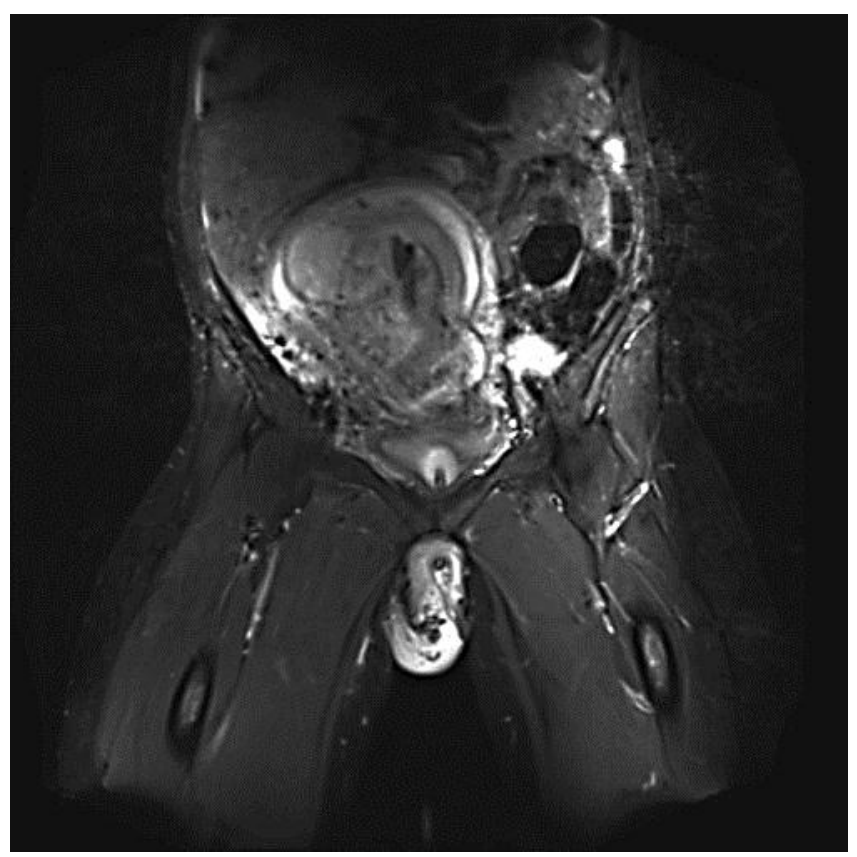

Fig. 1. MRI of the small pelvis: MRI scan performed in the 18th week of gestation showing the tumorous infiltrated uterus harboring the fetus.

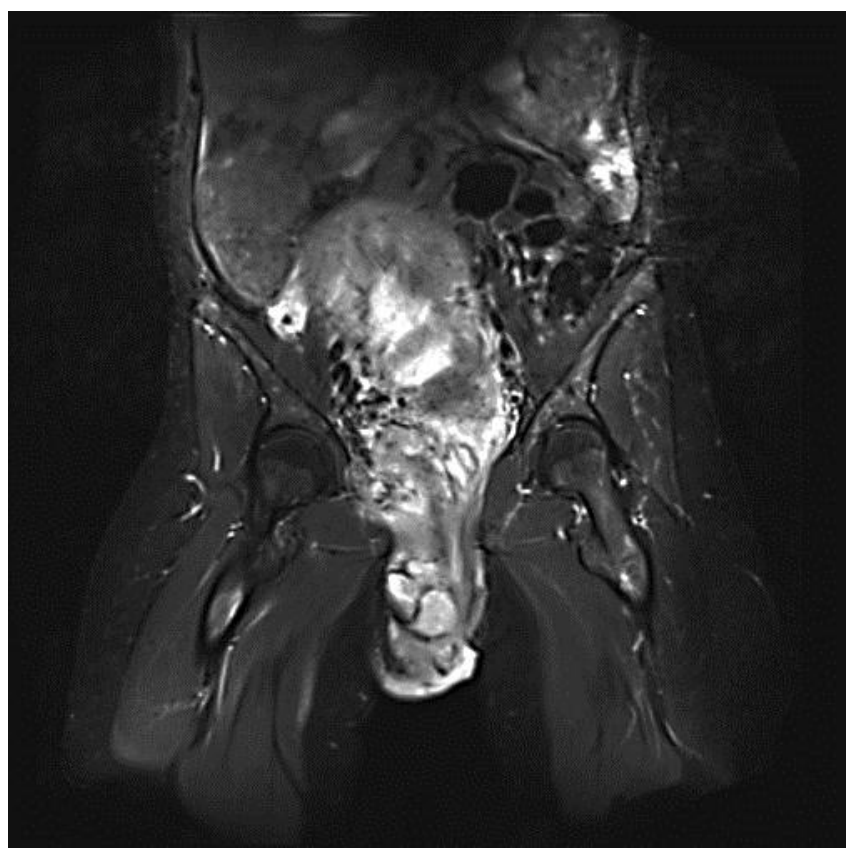

Fig. 2. MRI of the small pelvis: MRI scan performed in the 18th week of gestation showing the diffuse tumorous infiltration of the uterus, compression of the vagina, and dislocation of the cervix. 


\section{References}

1 Azim H, Peccatori F, Pavlidis N: Treatment of the pregnant mother with cancer: a systematic review on the use of cytotoxic, endocrine, targeted agents and immunotherapy during pregnancy. Part I: Solid tumors. Cancer Treat Rev 2010;36:101-109.

2 Brewer M, Kueck A, Runowicz C: Chemotherapy in pregnancy. Clin Obstet Gynecol 2011;54:602-618.

-3 Hogendoorn PC; ESM/EUROBONET Working Group; Athanasou N, Bielack S, De Alava E, Dei Tos AP, Ferrari S, Gelderblom H, Grimer R, Hall KS, Hassan B, Hogendoorn PC, Jurgens H, Paulussen M, Rozeman L, Taminiau AH, Whelan J, Vanel D: Bone sarcomas: ESMO Clinical Practice Guidelines for diagnosis, treatment and follow-up. Ann Oncol 2010;21:204-213.

-4 Merimsky 0, LeCesne A: Soft tissue and bone sarcoma in association with pregnancy. Acta Oncol 1998;37:721-727.

5 Jameel A, Jamil SN: Safety of cytotoxic chemotherapy during pregnancy. J Pak Med Assoc 2007;57:449452.

-6 Abellar RG, Pepperell JR, Greco D, Gundogan F, Kostadinov S, Schwartz J, et al: Effects of chemotherapy during pregnancy on placenta. Pediatr Dev Pathol 2009;12:35-41.

$\checkmark 7$ Meazza C, Casanova M, Zaffignani E, Clerici CA, Favini F, Vasquez R, Ferrari A: An adolescent with rhabdomyosarcoma during pregnancy. Tumori 2008;94:431-433.

-8 Aboujaoude R, Alvarez JR, Alvarez M, Al-Khan A: Follicular dendritic cell sarcoma in pregnancy: case report and review of the literature. Am J Perinatol 2006;23:459-461.

-9 Nakajima W, Ishida A, Takashi M, Hirayama M, Washino N, Ogawa M, et al: Good outcome for infant of mother treated with chemotherapy for Ewing sarcoma at 25 to 30 week's gestation. J Pediatr Hematol Oncol 2004;26:308-311.

10 Merimsky O, Le Chevalier T, Missenard G, Lepechoux C, Cojean-Zelek I, Mesurolle B, Le Cesne A: Management of cancer in pregnancy: a case of Ewing's sarcoma of the pelvis in the third trimester. Ann Oncol 1999;10:345-350.

11 Martin D, Winter SS, Gardner MO, Nicklaus P: Rhabdomyosarcoma treated with chemotherapy during the first trimester. Obstet Gynecol 1997;89:828-831.

12 Fernandez H, Diallo A, Baume D, Papiernik E: Anhydramnios and cessation of fetal growth in a pregnant mother with polychemotherapy during the second trimester. Prenat Diag 1989;9:681-682.

13 Lysyj A, Bergquist JR: Pregnancy complicated by sarcoma. Report of two cases. Obstet Gyencol 1963;21:506-509.

14 Haerr RW, Pratt AT: Multiagent chemotherapy for sarcoma diagnosed during pregnancy. Cancer 1985;56:1028-1033.

15 Rawlinson KF, Zubrow AB, Harris Ma, Jackson UC, Chao S: Disseminated Kaposi's sarcoma in pregnancy: a manifestation of acquired immune deficiency syndrome. Obstet Gynecol 1984;63:2S-6S.

16 Blatt J, Mulvihill JJ, Ziegler JL, Young RC, Poplack DG: Pregnancy outcome following cancer chemotherapy. Am J Med 1980;69:828-832.

$\checkmark 17$ Ateser G, Yildiz O, Leblebici C, Mandel NM, Unal F, Turna H, et al: Metastatic primitive neuroectodermal tumor of the ovary in pregnancy. Int J Gynecol Cancer 2007;17:266-269.

18 Mir O, Berrada N, Domont J, Cioffi A, Boulet B Terrier P, Bonvalot S, Trichot C, Lokiec F, LeCesne A: Doxorubicin and ifosfamide for high-grade sarcoma during pregnancy. Cancer Chemother Pharmacol 2012;69:357-367. 